\title{
MEMS-based Micro-Coriolis Density and Flow Measurement Technology
}

\author{
C. Huber \\ Endress+Hauser Flowtec AG, Kägenstrasse 7, CH-4153 Reinach, Switzerland \\ christof.huber@flowtec.endress.com
}

\begin{abstract}
Endress + Hauser has a long history of developing and producing flowmeters of various measurement technologies for the scope of process and product control in a variety of applications and industries. One of the most important flow measurement technologies in the last decades was the vibrating tube flowmeters based on the Coriolis measuring principle. An advantage of Coriolis flowmeters is the fact that they measure direct mass flow rate. Furthermore, by using the resonating tube frequency information, density and viscosity of a fluid can be deduced. Coriolis flowmeters exist in a lot of different shapes and styles, typically having metal flow tubes e.g. from stainless steel, titanium, zirconium or tantalum. The nominal flow rates range from about $1 \mathrm{~g} / \mathrm{h}$ to $4000 \mathrm{t} / \mathrm{h}$ corresponding to line sizes from $0.2 \mathrm{~mm}$ up to $350 \mathrm{~mm}$. In recent years different groups have worked on the adaptation and implementation of the Coriolis measuring principle in a MEMS (Micro Electro Mechanical System) sensor by etching a micro tube structure from a silicon wafer. Such micro machined silicon based Coriolis sensor chips open a new field of applications for the Coriolis measuring principle. Here we present the work of Endress+Hauser on the field of MEMS based Micro-Coriolis Sensors and explore potential applications of this new technology, e.g. for very accurate liquid and gas density measurements and concentration determination.
\end{abstract}

Key words: MEMS, micro-Coriolis, mass flow, density, viscosity

\section{Introduction}

Coriolis flowmeters have been one of the most important flow measuring principles of the last decades. It represents one of the fastest growing flowmeter markets [1]. A Coriolis flowmeter is seen as a very accurate measuring principle that is able to measure multiple parameters such as direct mass flow rate, temperature, density and even viscosity of the flowing fluid.

Endress+Hauser Flowtec AG is developing and producing Coriolis flowmeters since more than 20 years [2].

Coriolis flowmeters exist in a lot of different shapes and styles typically with metal flow tubes. There exist single tube, dual tube and even 4 tube meters. Flow tubes can be straight, bended or looped. It depends on specific industry specifications which meter will be considered. The nominal flow rates ranges from about $1 \mathrm{~g} / \mathrm{h}$ to $4000 \mathrm{t} / \mathrm{h}$ corresponding to line sizes from about $0.2 \mathrm{~mm}$ up to $350 \mathrm{~mm}$.
Another growing market is the micro fluidic market [3]. On one hand classical production processes of the chemical, medical or life science industry are miniaturized, on the other hand, classical laboratory sample analyzing and processing is done in-line or on a microchip level [4]. All these trends require measurement equipment for lower throughputs using less sample volume and having smaller meter dimensions.

In recent years different groups have worked on the adaptation and implementation of the Coriolis measuring principle in a MEMS sensor by etching a micro tube structure from a silicon wafer. Such micro machined silicon based Coriolis sensor chips allow now to consider microfluidic applications for the Coriolis measuring principle [5-13].

Some of these MEMS sensor designs have been mainly developed to measure density and viscosity [14-19]. Others are focusing on flow measurement at very-low flow rates $(10 \mathrm{mg} / \mathrm{h}$ to $100 \mathrm{~g} / \mathrm{h})[11-13]$. 


\section{MEMS Coriolis Chip}

In the last years Endress+Hauser has focused on the development of a robust process sensor for measuring density and concentration of gases and liquids using a MEMS Coriolis Chip as a sensing element. The MEMS Chip bases on the technology described by Sparks et al. [20].

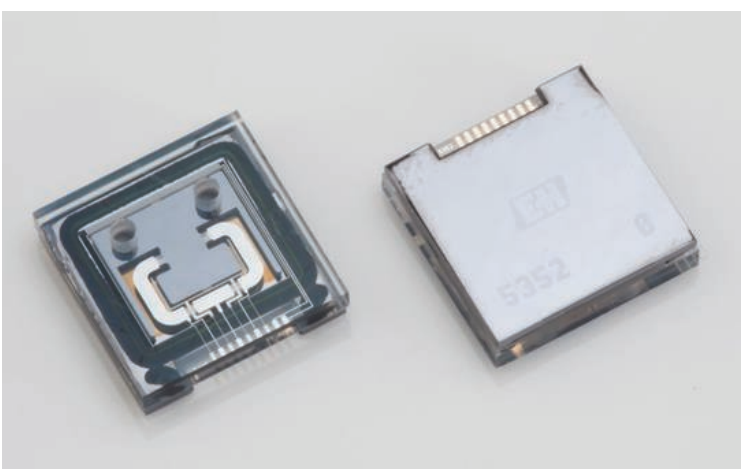

Fig. 1. E+H MEMS Coriolis sensor chip. Left: bottom view with fluidic connection holes, sensing and excitation pads. Right: top view on the sensor cap and the bond pads for electrical connections.

The resonant microtubes employs a MEMS fabrication process, which uses a combination of plasma and wet etching, photolithography, along with wafer bonding to form the microfluidic chips. Figure 1 shows a capped $\mathrm{E}+\mathrm{H}$ MEMS Coriolis sensor chip. It contains a freestanding omega shaped microtube that is able to resonate in a vacuum cavity.

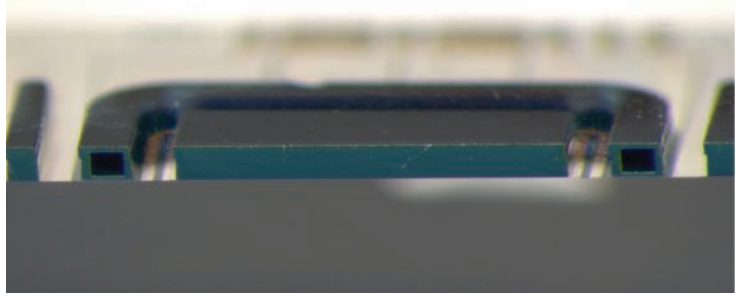

Fig. 2. Crosssection of the microtube

Figure 2 shows a crosssection of a microtube. The silicon tube is anodically bonded to a pyrex glass base plate. This glass wafer has the metal electrodes and runners used to carry electrical signals. The tube and the electrodes form three distinct capacitances. One is placed in the center of the tube and is used to excite the tube to resonate. The two other capacitors are placed in the inlet and in the outlet section of the tube, respectively. They are used to sense the tube deflection and are able to measure the resonance frequency of the tube which depends on the fluid density, as well as the phase shift between the inlet and the outlet signal which is a measure for the mass flow rate through the tube.

The metal layer is also used as an on-chip resistive temperature sensor. Two holes in the bottom glass chip admit fluid into and out of the silicon microtube (Fig. 1).

\section{MEMS Density Sensor}

The chip is able to measure mass flow rate [57] density [14-18] and even viscosity [19]. However, we focused on the industrialization of the density measurement.

A variety of density sensors are currently in use. For accurate readings (1 to $0.001 \mathrm{~kg} / \mathrm{m}^{3}$ ) density is most often measured using a resonating glass or metal tube. The density of a resonating tube depends on the stiffness $E^{\prime} l$, and the total mass of the tube $m_{\text {tube }}+m_{\text {fluid, }}$, according to eq. 1. Density measurement methodology is rather simple in such a device, the higher the weight of the fluid in the filled tube $m_{\text {fluid }}$, the lower the frequency $f$.

$$
f=\text { const } \sqrt{\frac{E \cdot I}{m_{\text {tube }}+m_{\text {fluid }}}}
$$

The mass of the fluid is proportional to the fluid density $\rho$ and the internal tube volume $V$ according to eq. 2 .

$m_{\text {fluid }}=\rho V$

With eq. 2 a relationship for the fluid density of the following structure can be found,

$\rho=A+\frac{B}{f^{2}}$

where $A$ and $B$ are calibration constants (depending on temperature and pressure).

The used silicon microtube has squared profile with dimensions of $200 \times 160 \mu \mathrm{m}$ and a overall length of about $15 \mathrm{~mm}$. Hence, the tube internal volume is only $500 \mathrm{nl}$. The tube resonates with frequencies between 20 to 30 $\mathrm{kHz}$. The small sample volume together with the high working frequency gives the potential for a very fast dynamic response of the sensor to density changes. The tube withstands pressures up to 80 bar.

Conventional density or coriolis flow sensors made from steel are well suited for liquid density measurements. But they are typically not sensitive enough to accurately measure gas density at low pressures. The big advantage of a silicon tube over a conventional steel tube is its 3.4 times lower material density, $m_{\text {tube }}$. This results in a significantly higher sensitivity of a 
silicon tube sensor for changes in the fluid density compared to a steel tube sensor with the same dimensions (eq. 1).

Hence, we can conclude a silicon microtube has several advantages compared to traditional density sensing technologies e.g. higher density sensitivity for gas at low pressures, smaller sample volume, higher vibration frequencies faster response time, smaller size and improved system integration.

\section{Integration into a Process Densitometer}

The MEMS chip requires electronics to amplify the sensor signals and drive the microtube. An electronic circuit is connected to the MEMS chip via wire bonds where the signal is processed to obtain the density and the temperature of the fluid flowing through the tube. The tube is electrostatically activated and its deflection is sensed by the change in capacitance of the sensor electrodes.

The MEMS chip is integrated in a fully developed process sensor with digital and analog communications possibilities. An intrinsic safe circuit enables the use of this technology with flammable liquids or in explosion hazardous atmospheres.
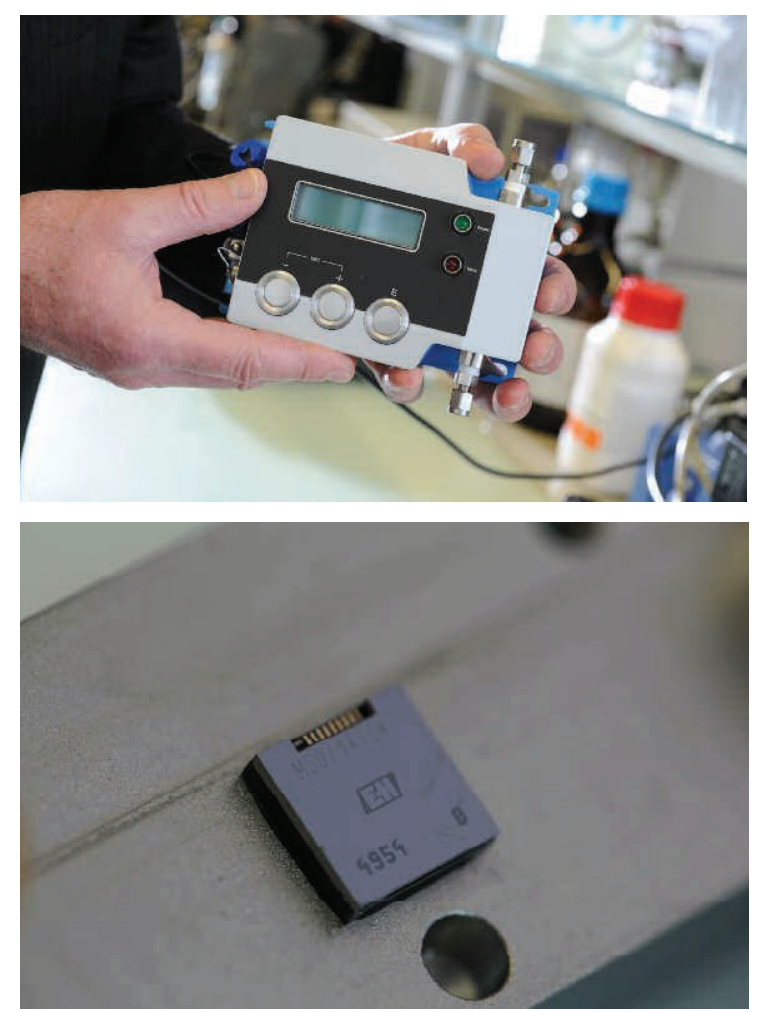

Fig. 3. Top: Process densitometer with fluidic connections, analog and digital communication used for the measurements shown in this paper. Bottom: MEMS sensor chip attached to a stainless steel manifold
The MEMS-chip is attached to a stainless steel manifold that can be connected to any process line using standard fluidic connections. One of the most important and difficult tasks on the way to a robust process sensor is the attachment of the MEMS chip to the metal manifold. A lot of experience is needed to evaluate the best solution for a specific application. We have a qualified solution that fits for technical non corrosive gases.

\section{Gas Density Measurements}

In order to accurately measure the density of a compressible gas using the frequency of a resonating tube requires the knowledge of the gas temperature and pressure. For this study a capacitive absolute pressure sensor was used in series with the density meter to measure the pressure.

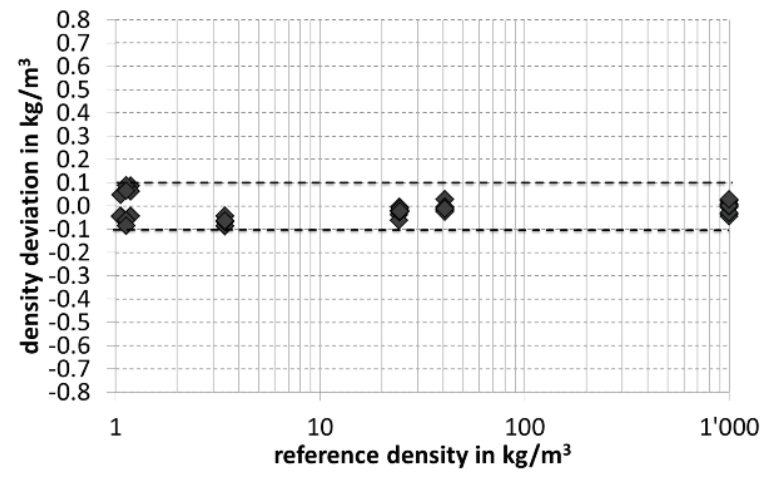

Fig. 4. Density calibration with nitrogen, carbon dioxide and water at different temperatures from 5 to $60^{\circ} \mathrm{C}$ and pressures from 1 to $20 \mathrm{bar}$

In Fig. 4 a typical gas calibration procedure is shown. Calibration fluids are nitrogen, carbon dioxide and water at different temperatures ranging from 5 to $60^{\circ} \mathrm{C}$ and pressures from 1 to 20 bar. The measuring accuracy for gases in this temperature and pressure range is \pm 0.1 $\mathrm{kg} / \mathrm{m}^{3}$.

Fig. 5 shows a comparison of the density measurements of several pure gases such as methane, nitrogen, argon, carbon dioxide and krypton versus pressure. Sensor signals were monitored continuously over a period time of about one hour. Starting from a working pressure of 20 bar the gas was slightly flowing through the sensor tube leading to a slow but steady decrease of the working pressure over time. The gases can be nicely distinguished by the density. 


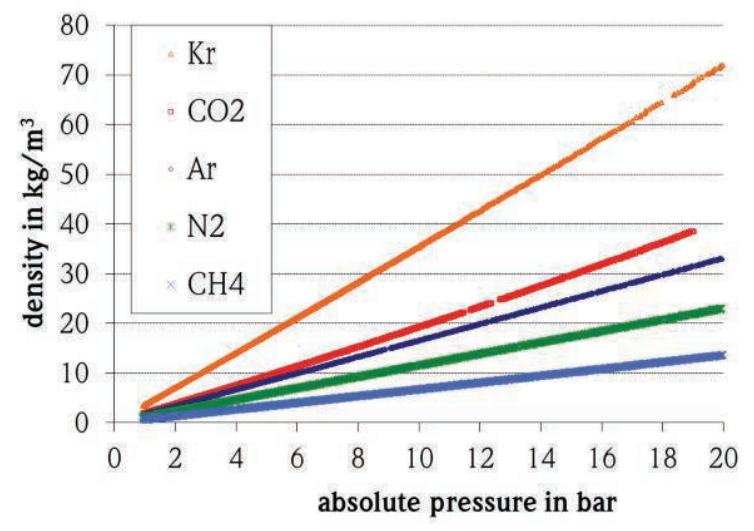

Fig. 5. Density measurements versus pressure of different pure gases.

In order to test the accuracy of the sensor, the measured density was compared to reference values taken from the NIST database [21]. The result for methane and nitrogen is shown in Fig. 6 . The sensor signal lies within an error band in the order of $\pm 0.1 \mathrm{~kg} / \mathrm{m}^{3}$. The repeatability is even better, about $\pm 0.05 \mathrm{~kg} / \mathrm{m}^{3}$.

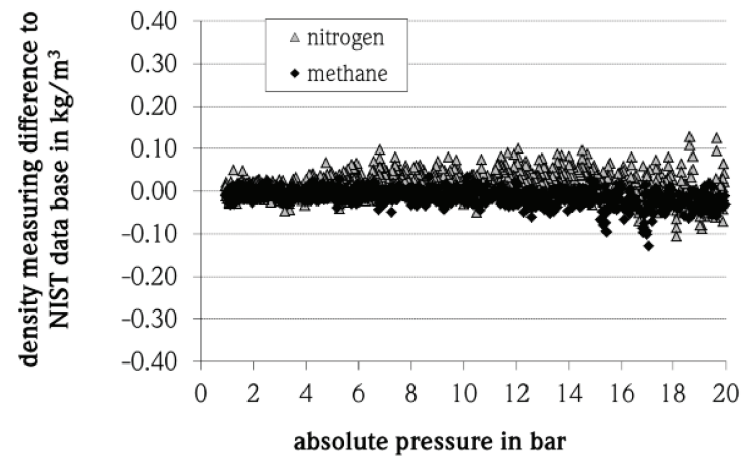

Fig. 6. Density measurements of methane (black dots) and nitrogen (gray triangles) versus pressure. Plotted is the density difference to the density values of the NIST database [21].

\section{Liquid Density Measurements}

Fig. 7 shows the measuring performance of the liquid calibration of two densitometers. A calibration was performed using nitrogen at different temperatures from 5 to $60^{\circ} \mathrm{C}$, water, IPA, different Cannon reference standards (N2, S6 and S20), tree different glycerol water mixtures $(50 \%, 60 \%$ and $80 \%$ glycerol $)$ and a sodium polytungstate water solution. The density and viscosity range of the liquid samples was 750 to $1350 \mathrm{~kg} / \mathrm{m}^{3}$ and 1 to 40 $\mathrm{mPas}$, respectively. The measured densities deviate within $\pm 0.25 \mathrm{~kg} / \mathrm{m}^{3}$ from the reference values.

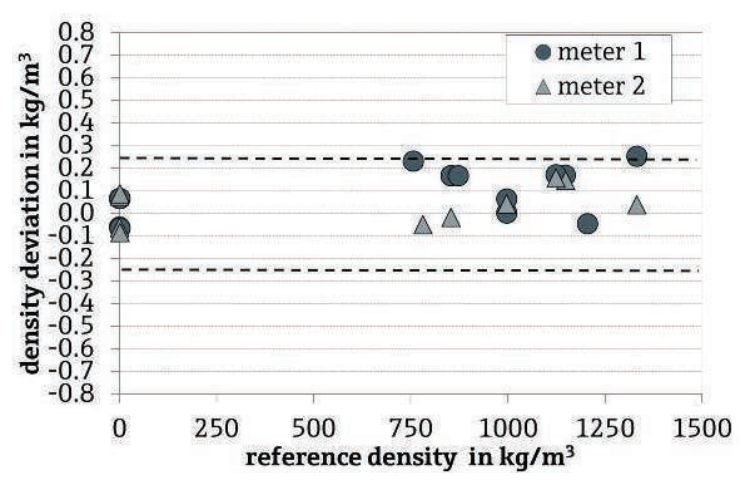

Fig. 7. Measurement with liquids of different densities and viscosities

In order to test the reproducibility of the sensor the density of different types of water was analyzed at room temperature. Deionized water, tap water from different sources and various bottled mineral waters were measured. The analyses were performed over a period of several days. Samples of 10 to $20 \mathrm{ml}$ were injected with a syringe one by one. The result can be seen in Fig 8. The densities of the different samples reproduce within less than $\pm 0.1 \mathrm{~kg} / \mathrm{m}^{3}$ over the whole time period. The reproducibility includes the uncertainty of the handling process and a potential contamination of the samples.

For the mineral water samples the density readings can be compared with the mineral content indicated on the bottles. Both values are strongly correlated. This shows the potential of the sensor to measure the total mineral content in water with an uncertainty of $100 \mathrm{mg} / \mathrm{L}$.

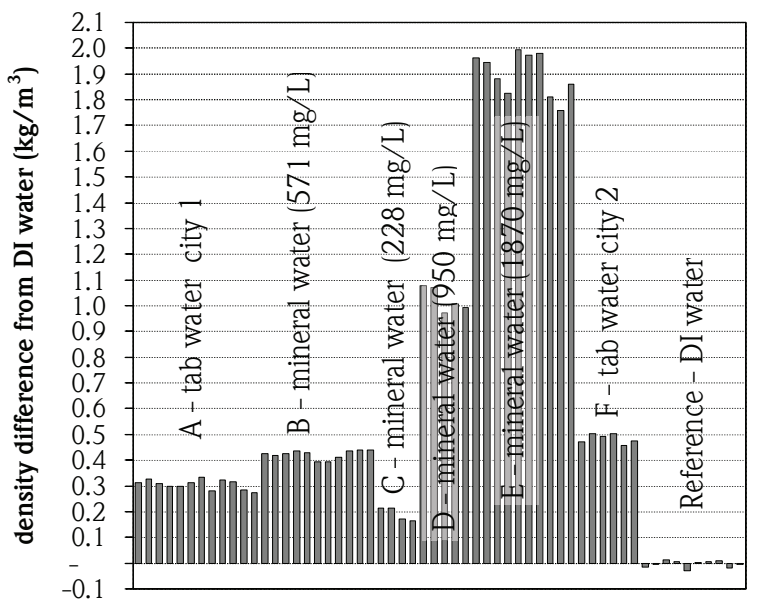

Fig. 8. Water density and total mineral content

\section{Conclusions}

The MEMS Coriolis sensor presented in this paper is representative for state-of-the art MEMS sensor development. Its small size, fast 
response time, vibration immunity and exceptional accuracy and reproducibility open up new possibilities for density and concentration measurement as a basis for gas and liquids quality control in a wide variety of applications and end user industries, both in process and laboratory environments. Industrialization and subsequent testing of this sensing technology has shown its capability for being applied in industrial environments.

\section{Acknowledgements}

The author would like to acknowledge the work and support from A. Rasch, P. Reith, H. Feth, S. Wirth, M. Theodor and M. Touzin.

\section{References}

[1] Wang T, Baker R. Coriolis flowmeters: a review of developments over the past 20 years, and an assessment of the state of the art and likely future directions. Flow Meas. Instrum. 40, 99-123. (2014); doi:10.1016/j.flowmeasinst.2014.08.015.

[2] Anklin M, Drahm W, Rieder A. Coriolis mass flowmeters: Overview of the current state of the art and latest research. Flow Meas. Instrum. 17(6), 317-323 (2006) doi:10.1016/j.flowmeasinst.2006.07.004.

[3] Flow Research Inc. The world market for Coriolis flowmeters ( $4^{\text {th }}$ ed.), 2013

[4] Castillo-Leon J, Lab on a Chip Devices and Micro-Total Analysis Systems, Springer Int. Publishing Switzerland, (2015), doi: 10.1007/978-3-319-08687-3_1

[5] Zhang Y, Tadigadapa S, Najafi N. A micromachined Coriolis-force-based mass flowmeter for direct mass flow and fluid density measurement. Transducer ' 01 Eurosensors XV, the 11th international conference on solid-state sensors and actuators. Munich, Germany (2001)

[6] Sparks D, Smith R, Cripe J, Schneider R, Najafi N. A portable MEMS Coriolis mass flow sensor. Proc. IEEE Sensors 2003 (IEEE Cat. No.03CH37498) 1(8),337-339 (2003) doi:10.1109/ICSENS.2003.1278953.

[7] R. Smith, D. Sparks, D. Riley, N. Najafi. A MEMS-based Coriolis Mass Flow Sensor for Industrial Applications, IEEE Transactions on Industrial Electronics, 56, 4, (2009)

[8] Haneveld J, Lammerink TSJ, de Boer MJ, et al. Modeling, design, fabrication and characterization of a micro Coriolis mass flow sensor. J. Micromechanics Microengineering 20(12):125001 (2010) doi:10.1088/0960-1317/20/12/125001.

[9] Clark C, Wang S, Cheesewright R. The performance characteristics of a micromachined Coriolis flow meter: An evaluation by simulation. Flow Meas. Instrum.
17(6):325-333 (2006)

doi:10.1016/j.flowmeasinst.2006.06.003.

[10] Sparreboom W, Geest J Van De, Katerberg $\mathrm{M}$, et al. Compact Mass Flow Meter Based on a Micro Coriolis Flow Sensor. Micromachines 22-33 (2013) doi:10.3390/mi4010022.

[11] Lötters JC, Lammerink TSJ, Groenesteijn J, Haneveld J, Wiegerink RJ. Integrated thermal and micro-Coriolis flow sensing system with a dynamic flow range of more than five decades. Micromachines 3, 194203 (2012)

[12] Wiegerink RJ, Lammerink TSJ, Groenesteijn J, Dijkstra M, Lotters JC. Micro Coriolis mass flow sensor for chemical micropropulsion systems. First international conference on microfluidic handling systems (MFHS-2012). Enschede, TheNetherlands (2012)

[13] P. Enoksson, G. Stemme, E. Stemme, A silicon resonant sensor structure for Coriolis mass-flow measurements, Journal of Microelectromechanical Systems 6, 119125. (1997)

[14] Sparks D., Smith R., Schneider R., Cripe J., Massoud-Ansari S., Chimbayo A., Najafi N., $A$ variable temperature, resonant density sensor made using an improved chip-level vacuum package, Sensors and Actuators $A$, 107, 119-124 (2003)

[15] Sparks D., Kawaguchi K., Yasuda M., Riley D., Cruz V., Tran N., Chimbayo A., Najafi N., Embedded MEMS based concentration sensor for fuel cell and biofuel applications, Sensors and Actuators A, vol.145- 146, 913 (2008)

[16] Sparks D., Goetzinger D., Riley D., Najafi N., A by-pass sensor package design enabling the use of microfluidics in high flow rate applications, Proceedings of the 2006 ASME MEMS/NEMS Packaging Symposium, Chicago, (2006)

[17] Sparks D., Smith R., Patel J., Nadjafi N., A MEMS-based low pressure, light gas density and binary concentration sensor, Sensors and Actuators, A171, 159-162 (2011)

[18] Huber C., Touzin, M, New MEMS-based micro-coriolis density measurement technology, Proceedings of the ASME 2011 9th International Conference on Nanochannels, Microchannels, and Minichannels, Edmonton, Alberta, CANADA, (2011) ICNMM2011-58030

[19] Sparks D., Smith R., Cruz V., Chimbayo A. , Riley D., Najafi N., Dynamic and kinematic viscosity measurements with a resonating microtube, Sensor and Actors A , 149, 3841 (2009) 
[20] D. Sparks, R. Smith, N. Najafi, Microfluidic Device and Method of Operation, Patent US7581429 B2, (2009)

[21] NIST National Institute of Standards and Technology, Thermophysical Properties of Fluid Systems,

http://webbook.nist.gov/chemistry/fluid/ 